kreise Spree-Neiße (+ 106\%), Uckermark $(+102,7 \%)$ sowie Coburg (+89,4\%) vergleichsweise die höchsten Werte. Wie es zu diesen teilweise horrenden Divergenzen kommt, vermögen die Experten nicht zu erklären.

Zu den Unterschieden auf Landesebene meinte Bruckenberger: „Als Gründe für die zum Teil deutlich voneinander abweichenden Sterbeziffern in den einzelnen Bundesländern werden u.a. die unterschiedlichen Lebensweisen aufgrund der sozialen Situation mit Zigarettenkonsum, Fettleibigkeit und Bewegungsmangel, aber auch Schnelligkeit, Qualität und Organisation des Rettungsdienstes vermutet. Doch gibt es auch in Regionen mit geringer Arbeitslosigkeit teilweise überdurchschnittliche Sterbeziffern, sodass wir letztlich über die Ursachen nur spekulieren können.“

\section{Schweiz wieder spitze}

Wie schon in den letzten Jahren liegt Deutschland im Drei-Länder-Vergleich mit Österreich und der Schweiz sowohl bei der stationären Morbiditäts- und der Sterbeziffer als auch bei der Häufigkeit der Linksherzkatheterunter- suchungen, der PCls und der Herzoperationen teilweise deutlich vor den Vergleichswerten aus Österreich und der Schweiz, wobei die Schweiz den Spitzenplatz in diesem Ländervergleich seit Jahren imposant verteidigt.

Ein Ergebnis, das betroffen macht und bislang ohne plausible Erklärung bleibt. Jedenfalls kann nicht behauptet werden, dass das schlechte Abschneiden Deutschlands in diesem Vergleich an zu wenigen Behandlungsmöglichkeiten liegt. Im internationalen Vergleich belegt Deutschland bei den Herzkatheterlabors einen Spitzenplatz.

\section{Mehr Ablationen}

Weiter im Aufwärtstrend sind auch die elektrophysiologischen Untersuchungen (EPUs) und Ablationen. Die DGK meldete insgesamt 51496 EPUs und 40815 Ablationen in ganz Deutschland, wobei in dieser Auflistung unerfindlicherweise die Zahlen aus Bremen fehlen. Im Bundesdurchschnitt waren es 630 EPUs und 499 Ablationen pro 1 Mio Einwohner. Die Spannbreite pro 1 Mio Einwohner reichte bei den EPUs von 241 (Schleswig-Holstein) bis 1630 (in Hamburg), bei den Ablationen von 164 (Schleswig-Holstein) bis 1600 (in Hamburg). Gegenüber dem Vorjahr ist bei den EPUs ein Anstieg von $17,9 \%$ und bei den Ablationen um 18,3\% zu registrieren.

\section{Chest Pain Units}

In Deutschland gab es Anfang Oktober knapp 100 zertifizierte Chest Pain Units (CPU). Wie der Pionier dieser DGK-Initiative, Prof.Münzel, Mainz, ausführte, beginnt im nächsten Jahr bereits der Prozess der Rezertifizierung. Bisherige Daten aus Deutschland, den USA und England belegen, dass die Organisationsmodelle einer CPU neben einer signifikanten Reduktion der Infarktmortalität auch zu einer Verkürzung der durchschnittlichen Liegedauer und einer Kostenreduktion durch rationelle Diagnostik und Therapie führen. In Deutschland, das in der Etablierung von CPUs inzwischen führend in Europa ist, fällt allerdings auf, dass sich die CPUs v.a. in Ballungszentren befinden, nicht aber in mangelhaft versorgten Regionen, die von einer CPU besonders profitieren würden.

Dr. med. Jochen Aumiller I

Kommentar von Prof. Friedhelm Beyersdorf, Freiburg

\title{
Alarmierend: Die Zahl der Herztransplantationen sinkt
}

„Dem neuen Herzbericht sind fünf wesentliche Trends zu entnehmen:

- Zunehmende Zahl von Arterien als Bypassmaterial bei der Koronarrevaskularisation,

- Weiterentwicklung der Technologie der Herz-LungenMaschine bei einem immer schonenderen Einsatz, - exzellente Betreuung der Patienten nach der Operation auf herzchirurgischen Intensivstationen (hoher Anteil an Intensivbetten an der Gesamtbettenzahl im Vergleich mit anderen Fächern),

- gleichbleibend hervorragende chirurgische Ergebnisse trotz deutlich gestiegenen Risikoprofils, insbesondere drastisch zunehmenden Alters der Patienten,

- zunehmender Einsatz von Herzunterstützungssystemen bei sinkendem Transplantationsaufkommen.

Der ,Herzbericht 2009' zeigt, dass die Gesamtleistungen der herzchirurgischen Zentren in Deutschland im Vergleich zu 2008 nochmals um 3000 Eingriffe gesteigert werden konnten (die Gesamtoperationszahlen stiegen von 159205 auf 162417 an). Während in der Koronarchirurgie ein Abfall von ca. 2500 Operationen zu verzeichnen war, gab es eine Steigerung im Bereich der Klappenchirurgie, der thorakalen Aortenchirurgie und des Einsatzes von Herzunterstützungssystemen. Im Bereich der extrakardialen Eingriffe war eine Steigerung von ca. 3500 Eingriffen zu verzeichnen.

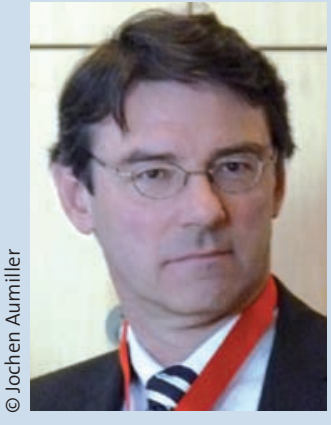

Prof. F. Beyersdorf, Präsident des Herzchirurgenverbands DGTHG einen erneuten Rückgang des Trans-
plantationsaufkommens in Höhe von $5 \%$ gegenüber dem Vorjahr. Inzwischen wird die Wartelistensituation für eine Herzverpflanzung immer schwieriger: Bereits 80 bis $90 \%$ der transplantierten Patienten stehen auf der höchsten Dringlichkeitsstufe (High urgency), bei ihnen besteht aufgrund einer akut lebensbedrohlichen Situation eine besondere Dringlichkeit zur Transplantation. Sie werden vorrangig vor allen anderen Patienten transplantiert. Eine Verbesserung der Situation ist v.a. durch eine Aufstockung der Koordinatorenpositionen in den Krankenhäusern zu erreichen." 